\title{
EN TORNO A EL ORIGEN DE LA OBRA DE ARTE DE MARTIN HEIDEGGER ${ }^{1}$
}

\author{
François Fédier ${ }^{2}$
}

\begin{abstract}
Resumen / Abstract
Es necesario distinguir entre útil, cosa y obra de arte. Lo que nos circunda en la óptica de un "para" apunta al conjunto de los útiles. La cosa es aquello de lo que se habla. Aunque las relaciones entre útil y cosa son estrechas, es posible hablar de las cosas que nos circundan desde perspectivas que no se reducen a la óptica del "para". Una cosa no se reduce a ser para algo. Una obra tiene un tipo de presencia singular; ella no está ahí sino para aquellos que, por su lado, están expresamente abiertos a ella. El origen de la obra de arte es la verdad. Ésta no tiene que entenderse como la adecuación de lo que nosotros decimos a lo que es en realidad. Heidegger invita a escuchar el nombre griego de la verdad-alétheia. 'A $\lambda \hat{n} \theta \varepsilon ı \alpha$ [alétheia] hace reparar en el alfa privativa y el tema que deriva del verbo $\lambda \alpha v \theta \alpha ́ v \omega$ [lantháno]: escapar.
\end{abstract}

Palabras clave: Heidegger, origen, obra de arte, cosa, útil, verdad como alétheia.

\section{ON HEIDEGGER's THE ORIGIN OF THE WORK OF ART}

It is necessary to distinguish between useful thing, thing and work of art. What surrounds us in the optics of a "for" points at the totality of useful things. Thing is what we talk about. Even though the relationships between useful thing and thing are narrow, it is possible to talk about the things that surround from perspectives that are not restricted to the optics of "for". A thing is not restricted to be for something. A work of art has a singular type of presence; it is not there but for those who, on their side, are expressly open to her. The origin of the work of art is the truth. It must not be understood as the adequacy of what we say to what it is in reality. Heidegger invites to listen to the Greek name of truth -alétheia. A $\lambda \dot{\eta} \theta \varepsilon i \alpha$ [alétheia] makes you pay attention to the privative alpha and the theme deriving from the verb $\lambda \alpha v \theta \dot{\alpha} v \omega$ [lantháno]: escape.

KEY WORDS: Heidegger, origin, work of art, thing, useful thing, truth as alétheia.

En L'art en liberté, Pocket, París, 2006; pp. 295-309. Es el texto empleado por el autor en una conversación organizada por Didier Laroque el 23 de marzo de 2005 en el Museo de Bellas Artes de Orléans. El resumen y las notas son del traductor.

2 François Fédier es filósofo. Nació en 1935. Ha sido discípulo de Jean Beaufret desde 1955. Se consagra, a partir de 1958, a la traducción de textos de Heidegger. Dirige la traducción, en curso, de la Edición Integral (Gesamtausgabe) de Heidegger en la editorial Gallimard. Ha sido docente en primera superior (Khâgne) en el liceo Louis Pasteur (Neuilly-sur-Seine), hasta su retiro en 2001. 
$\overline{R H}$ Vamos a intentar hablar de la conferencia que redactó Martin Heidegger en 1935 bajo el título El Origen de la obra de arte. Hablar así a propósito de algo es exponerse, siempre, al riesgo de caer en el parloteo. Uno de los parapetos que nos puede preservar de ello consiste en hablar juntos acerca de ese algo. Por eso empleo ese "nos", que no es un plural de majestuosidad sino que con él se expresa mi entrañable deseo de que, viniendo esta tarde ante ustedes, la palabra no se quede en un único sentido. Lo sé bien: habitualmente, esperar que vengan preguntas de un auditorio, más que suscitar el aflujo de preguntas, lo bloquea. También voy a intentar decir algunas cosas que, así lo espero, puedan disponernos a interrogarnos en conjunto.

El Origen de la obra de arte es el primer texto del libro Caminos / que no llevan a ninguna parte. El título de este libro es, en alemán, Holzwege. Wege son los caminos. Holz es el bosque. René Char nos ha hecho un día el reproche de no haber dado al libro, en su versión francesa, el título literal: Caminos del bosque.

En cierto sentido, él tiene razón, indiscutiblemente. Pero, por otro lado, no se puede ocultar que en alemán corriente la palabra Holzweg hace pensar, indefectiblemente, en la locución "auf dem Holzweg sein", mediante la cual se alude al hecho eminentemente penoso de estar en un callejón sin salida-y, más aún, de estar bloqueado en un atolladero, de no saber ya cómo librarse para salir de él.

Me acuerdo que preparando la edición de esta traducción, he dado con un poema de Rainer Maria Rilke que forma parte de las Cuartetos valaisanos. Rilke ha escrito en francés estos Cuartetos, alrededor de los años 20.

Chemins qui ne mènent nulle part

entre deux prés,

que l'on dirait avec art

de leur but détournés,

chemins que souvent n'ont

devant eux rien d'autre en face

que le pur espace

et la saison.

Caminos que no llevan a ninguna parte

entre dos prados

que, con arte se lo diría,

de su meta desviados,

caminos que a menudo no tienen

ante ellos nada más que, enfrente,

el puro espacio y la estación ${ }^{3}$.

3 Véase, Poemas franceses, Ed. Pre-Textos, Madrid, 1997, p. 117. Ed. bilingüe. Trad. de Tomás Segovia. Acojo esta traducción con ligeras variaciones. 
Que Heidegger haya elegido denominar Holzwege 4 el primer libro que publicó después de más de veinte años de ausencia editorial es, con seguridad, un indicio al que conviene prestar un poco de atención. Indicio, ante todo, del hecho de que él sabe que ha tomado un itinerario muy insólito -pudiendo dar a más de uno la impresión de haber partido hacia regiones poco frecuentadas, de haberse perdido en ellas, si es que no, inclusive, de haberse extraviado inextricablemente en una especie de arenas movedizas.

Ahora bien, lo que dice aquí Rilke describe de maravilla aquello delante de lo cual ha llevado el camino seguido por Heidegger: el espacio puro y la estación -nada tangible, pero, sin embargo, algo en el seno de lo cual todo lo que se puede tocar necesariamente viene a tomar algún lugar.

Como he dicho al comienzo, El Origen de la obra de arte data de 1935, es decir, del momento en que Heidegger ha comenzado de veras ese movimiento de regreso río arriba, que, cabalmente bien considerado, no es sino una vuelta hacia la cúspide, en la medida en que se interna en otra parte, otra parte en la que la distinción entre lo alto y lo bajo ya no puede proporcionar las marcas geodésicas de una partición espacial.

El Origen de la obra de arte es, así, antes que nada, un texto en el que Heidegger alcanza una especie de "punto de no-retorno". Habría mucho que decir sobre esta idea del punto de no-retorno. Veo en ella en primera instancia algo así como una figura emblemática en la que es la existencia humana como tal quien toma un relieve que deja pasmado.

¿Por qué - a propósito de esto- se pone en cuestión la obra de arte? Intentemos encontrar la respuesta considerando el texto, comenzando por ahí donde Heidegger pone en relación la obra [œuvre], con la cosa [chose], por una parte, y con el producto [produit], por otra parte -al menos es así como se lo puede leer en la traducción francesas de este texto 5 .

Desde ahora precisemos exactamente lo que está en cuestión; para eso, volvamos a la formulación original, en lengua alemana; no porque el alemán sea una lengua privilegiada - están bien persuadidos de eso-, sino, muy simplemente, porque ninguna lengua habla completamente como otra.

Obra se dice en alemán das Werk (como en inglés se dice work-pero hay una ligera diferencia de registro entre el inglés y el alemán).

Cosa se dice en alemán das Ding (como en inglés se dice a thing).

Producto se dice en alemán das Zeug.

4 Véase, ahora, Gesamtausgabe (Edición Integral), Vol. 5: Holzwege, V. Klostermann, Frankfurt a. M., 1977. Ed. de Friedrich-Wilhelm von Herrmann. Primera ed.: 1950.

5 “L'origine de l'œuvre d'art". En Chemins qui ne mènent nulle part, Gallimard, París, 1962. Trad. de Wolfgang Brokmeier. Edición de François Fédier. Versión en español, en Caminos de bosque, Ed. Alianza, Madrid, 1995. 10ª ed., 2010. Trad. de Helena Cortés y Arturo Leyte. Otra versión se debe a Francisco Soler Grima; ha sido recogida en su libro El origen de la obra de arte y la verdad en Heidegger, Universidad Nacional de Colombia, Bogotá, 1953. 
Aquí, a propósito de este último término, conviene detenerse un momento. Porque das Zeug es en alemán una palabra singular (en todos los sentidos del término, y, ante todo, porque es una palabra que no tiene plural). Eso plantea un terrible problema de traducción. El traductor, Wolfgang Brokmeier -en ese tiempo, un joven alemán que había pasado la mayor parte de su infancia y de su adolescencia en Francia-se resignó a verter Zeug por producto. ¿Por qué digo que "se resignó"? Porque eligiendo esta solución perdía contacto con todo un lado del análisis del Zeug, precisamente, análisis efectuado por Heidegger en Ser y Tiempo, el libro clave de $1927^{6}$.

Voy, pues, a exponer a grandes rasgos lo que este análisis tiene de esclarecedor para nuestro propósito.

Abandonemos desde la partida la referencia a la lengua alemana e instalémonos en medio de los recursos de nuestra lengua.

Lo que designa directamente, en alemán, la palabra Zeug es lo que podemos hacer que se entienda en castellano recurriendo a la paráfrasis: lo que nos circunda, donde quiera que miremos, en la óptica de un "para".

Así, la sala donde nos encontramos, si la aprehendemos (inclusive sin decírnoslo explícitamente) como lugar para hacer, por ejemplo, conferencias. O bien la hoja de papel que tengo bajo los ojos, y que veo, de entrada, como lo que sirve para anotar lo que intento explicar. O bien, además, la línea de ferrocarril París-Orléans: para unir rápida y cómodamente las dos ciudades. Esta pluma: para escribir. La ventana: para dejar entrar la luz. Se podría continuar indefinidamente dando ejemplos, porque esta óptica en la que se ve lo que es como siendo para..., esta óptica, digo, nos es casi congénita. Es una de las caras capitales bajo las cuales nos aparece lo que nos circunda.

No es que todo nos aparezca necesariamente en esta óptica. Es aquí donde comenzamos a entrever la razón de la distinción que constituye el comienzo de la conferencia de Heidegger.

Nuestra palabra "cosa" -lo sabemos bien- nos viene derivada de causa, una palabra típicamente latina, cuya etimología es desconocida, pero cuyo sentido es perfectamente patente: es aquello que está en cuestión (y especialmente en un proceso: aquello de lo que se debate). La cosa es: aquello de lo que se habla. En este momento les hablo de El Origen de la obra de arte, en el marco de una conversación. Importa poco que la palabra alemana das Ding no cubra exactamente la acepción de nuestra palabra. Es posible hablar de las cosas que nos circundan desde perspectivas que no se reducen a la óptica del "para”. ¿Hay, inclusive, una óptica específica que corresponda a la manera en que nos aparecen las cosas? Dejemos la pregunta abierta. Lo esencial por el momento es que aquello que es puede aparecer también como cosa [chose], y que una cosa no se reduce a ser para algo [pour quelque chose]. Como dicen los filósofos, una cosa no es comprendida, primeramente, en el seno de una finalidad.

6 En castellano: Ser y Tiempo, Ed. Universitaria, Santiago de Chile, 1997. 5 ed., 2015. Trad. de Jorge Eduardo Rivera Cruchaga. 
Pensar que el Loira fluye para irrigar el campo, que el árbol en el jardín crece para dar sombra, o bien que el melón presenta hendiduras para ser comido más fácilmente en familia, eso nos parece, con toda razón, un poco ingenuo, o más exactamente: demasiado pueril.

Pero, en cambio, no es pueril en modo alguno -al contrario-, calcular por ejemplo la estructura de un puente para atravesar un valle profundo, que es el objetivo de un trabajo muy complejo de ingenieros altamente especializados que ponen en juego saberes muy reales. Si hay, incuestionablemente, cierta ingenuidad en imaginar una finalidad que gobierna discretamente todo lo que nos circunda, todo el trabajo humano, sin embargo, está orientado por una finalidad, hasta el punto, inclusive, que cuanto más lo sea, ese trabajo tiene más oportunidades de efectuarse con eficacia y éxito.

Eso es lo que hace resaltar la distinción entre "cosa" y "producto", distinción que hallamos en el comienzo de la conferencia. Recupero la palabra producto; en primer lugar, porque es la palabra que encontrarán en la traducción francesa; pero también, para mostrar fehacientemente que esta traducción no es en modo alguno aberrante. Porque lo que es producido, es sobre todo lo que es "producido para" tal o cual uso ["faite pour" tel ou tel usage], como lo dice tan bien la locución. Notemos, sin embargo, que puede haber muy bien tal finalidad sinfabricación, en el sentido estricto del término. Agrego esta advertencia adrede, porque el objetivo de Heidegger -cuando distingue la "cosa" del "producto"- no consiste tanto en oponer un mundo natural a un mundo humano. Es, mucho más finamente, distinguir entre dos perspectivas de sentido: en primer lugar, ésa donde el sentido es lo más inmediatamente articulado gracias a la utilidad (donde los productos del ingenio humano proporcionan, por así decirlo, espontáneamente los ejemplos de referencia); en seguida, ésa donde el sentido se articula con el lenguaje, el cual es mucho más diversificado que la utilidad. De paso, aprovecho la ocasión de recordar que en la traducción de Ser y Tiempo que ha proporcionado François Vezin, y que ha aparecido en la editorial Gallimard7, la palabra Zeug no es traducida por "produit" ["producto"], sino por "util" [u-t-i-l; pronúnciese: util-para distinguirlo del outil ${ }^{8}$, que es algo más restringido que el género más amplio de los utils [útiles]. El outil es un utensilio. El util puede ser algo diferente de un utensilio. En términos lógicos: todos los outils son utils; pero no todos los utils son, necesariamente, outils].

7 Etre et Temps, Gallimard, París, 1986. En las pp. 546 ss., Vezin redacta una nota, con la colaboración de W. Brokmeier, para explicar por qué traduce Zeug por util (primera nota a la p. 68 de la edición en alemán, paginación que aparece en los márgenes de la versión francesa).

8 Rudolf Boehm y Alphonse de Waelhens traducían Zeug por outil. Cfr., L'Être et le Temps, Gallimard, París, 1966, § 15. Véanse pág. 92 y pág. 289 , 2 ${ }^{a}$ nota a la p. 68 (de la edición en alemán). En este último lugar los traductores advierten "que se debe hacer notar que la palabra "outil" se toma aquí en el sentido amplio de "objeto de uso", y no solamente en el de "instrumento". Sin embargo, la mayor parte de los ejemplos citados a continuación conciernen a instrumentos. 
Util no es una invención del traductor, sino una palabra de la que Montaigne, por ejemplo, hacía uso, y que designa, simplemente, todo aquello que es útil [utile], todo aquello que aparece claramente como siendo para..., es decir, al servicio de algo. Así, en alguna parte de Ser y Tiempo dice Heidegger que un camino es un util (mientras que decir que es un outil chocaría con el espíritu de la lengua francesa). A partir de tales ejemplos se puede ver la calidad de una traducción reflexiva, es decir, de una traducción que se empeña en ponerse a reflexionar sobre sí misma.

Efectuada esta primera distinción, ya es hora de interrogarnos sobre el tercer término: la obra de arte. ¿Qué viene a hacer aquí la obra de arte? Déjenme dar un salto para ganar tiempo. Haciendo esto resumo un desarrollo que, sin embargo, se efectúa -a propósito-, paso a paso. Por eso será preciso evitar entender lo que acabo de decir como una declaración dogmática; hay que tomarlo, más bien, como invitación a plantearse preguntas.

Lo que acaba por exponer Heidegger es que muy posiblemente no estaríamos en condiciones de hacer la distinción entre cosa y útil si no hubiese obras. Tal distinción, repito, no es del todo simple, ni, sobre todo, fácil de pensar. Lo recién enunciado, dicho de esa manera, es casi incomprensible. Por tanto, vamos a intentar avanzar de otra manera.

¿Qué pasa con una obra de arte? Entendamos bien: ¿después de haber puesto al descubierto - tan poco como haya sido- lo que pasa, desde el punto de vista del sentido, con una cosa y con un útil, preguntamos lo que pasa con una obra? Para decirlo un poco apretadamente: estando frente a una obra, no estamos ya ni en el mundo de las cosas, ni en el mundo de los útiles. Si esto es así, se trata de algo que no descubrimos de entrada, como ocurre en el mundo del útil -gracias a la evidencia, en este último caso, que se manifiesta apenas captamos para qué está hecho. ¡Tampoco nos ayuda extendernos acerca de esto -inclusive, y sobre todo, si nuestro objetivo es llegar a decir algo esencial!

La presencia de una obra nos pone a quienes la tomamos en consideración en otra parte respecto de donde estamos corrientemente. Esta es una observación que no es nueva, pero que debemos examinar con toda la seriedad que merece.

Cuando Matisse declara, por ejemplo: "No trabajo sobre el lienzo, sino sobre aquel que lo mira", su declaración no es menos desconcertante que lo que acabamos de afirmar. Porque, medida con el mismo rasero de lo que nos parece obvio en el mundo habitual, casi no tiene sentido hablar como lo hace Matisse (en la medida en que ningún pintor ha pintado "realmente" una obra sobre un espectador). En situaciones de este tipo, recurrimos a la escapatoria que consiste en tachar de "metáfora" tal manera de hablar.

Pero, más que hablar como se habla habitualmente, tomémosles la palabra a las cosas, y digamos: el mundo de la obra es un verdadero mundo, como lo es el mundo en que vivimos habitualmente. La única diferencia con este mundo consiste en que no estamos en él sino de manera efímera, por poco tiempo cada vez. Hay, pues, una diferencia de tiempo. En el mundo de las cosas estamos "todo el tiempo" ["tout le temps"] -mientras que en el mundo de la obra estamos solamente de cuando en cuando [de temps en temps]. 
Una nueva declaración desconcertante. Pero ¿está permitido siquiera hablar así? Con la condición, tal vez, de que precisemos que por "mundo" es preciso no entender subrepticiamente el conjunto de las cosas -dicho de otro modo, aquello que los doctos llaman el universo.

Resumamos, pues, para estar en condiciones de avanzar. Resumir, aquí, es reunir en orden lo que hemos visto. Establezcamos esto: el término mundo debe ser entendido aquí como designando un conjunto que presenta una unidad característica. Hay así un mundo de la utilidad cuando hay una totalidad de útiles. Este mundo es, de alguna manera, la matriz donde se experimenta primordialmente la idea de conjunto. De este hecho, a partir de la idea de sentido, que ilustra con toda claridad la utilidad (ser-útil, es decir, ser como hecho para...), se pasa a la idea global de sentido, entendido ahora no como dirección sino como significación; de modo que el conjunto de todo aquello de lo que se puede hablar, de manera más o menos sensata, es el conjunto de lo real. De donde se ve que las relaciones entre útil y cosa son estrechas. La cosa aparece así como lo que es, inclusive al margen de toda utilidad. Esto, por lo demás, concuerda con la observación hecha antes sobre el tiempo.

Mientras que el carácter de utilidad viene a primer plano mientras se hace uso de un útil [de una manera, por lo demás, extraordinariamente sorprendente, puesto que la utilidad de mi pluma penetra completamente en el acto de escribir mientras escribo con ella, hasta el punto de unificarse con él], ocurre, frente a eso, que el carácter de cosa toma un aspecto de permanencia inalterable, una permanencia que tiende a aparecer como independiente de nuestra relación con las cosas. Es esta permanencia lo que llega a ser, así, el carácter más manifiesto del mundo de las cosas. En lo sucesivo, solo el mundo de las cosas se admite como el único verdadero mundo - hasta el punto de que se tiene, espontáneamente, la ilusión de no hablar ya sino metafóricamente desde que se menciona otro mundo diferente al de las cosas.

Esto llega a ser particularmente impresionante desde que se es confrontado con las obras. Constantemente, somos tentados a tomar a estas últimas -por cierto lado- como cosas que se pueden encontrar entre otras cosas en alguna parte dentro del seno del mundo habitual. No obstante -ya lo hemos señalado antes- un indicio muy sorprendente debería habernos alertado: una obra tiene un tipo de presencia completamente singular, en la medida en que ella no está ahí sino para aquellos que, por su lado, están expresamente abiertos a ella. Si observamos bien, es exactamente lo que dice la declaración de Henri Matisse que hemos citado.

"No trabajo sobre el lienzo, sino sobre aquel que lo mira".

¿Esto es decir que el pintor no da pinceladas de color sobre un lienzo? Con seguridad, sí las da. ¡Pero ese trabajo no es su verdadero trabajo! Trabajar como pintor no es trabajar como artesano. Esta advertencia nos debe incitar a ser, del todo, particularmente circunspectos - por poco que nos recordemos de lo que dice Heidegger en otro texto que la conferencia que nos retiene en este momento. Los griegos, escribe, 
no distinguen el arte de la artesanía, no teniendo para los dos sino un único nombre,

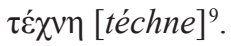

¿Esto quiere decir que comprendían implícitamente el arte a partir de la artesanía? O bien, a la inversa, ¿la artesanía a partir del arte? O bien, aún, ¿algo diferente? Hay poderosos motivos para apostar que es por el lado de la tercera posibilidad por donde es preciso indagar.

¿Cuál es la acepción griega de $\tau \dot{\varepsilon} \chi v \eta$ [téchne]? A propósito de esto, Heidegger da una indicación preciosa. En griego, esta palabra significa que se es entendido en lo que se hace. "Hacer", aquí, debe ser tomado en la significación precisa de hacer ser, hacer venir a ser. Y ahí palpamos, por así decirlo, la razón por la cual en Grecia no hay confusión al no distinguir entre artesanía y arte. Porque el ebanista que trabaja como carpintero una mesa, tanto como el escultor que moldea una estatua, hacen, cada uno, venir a ser algo que no estaba antes que ellos, ambos, comenzaran su trabajo. Porque cada uno trabaja ayudándose de todos los conocimientos que han podido acumular -no siendo estos conocimientos del todo teóricos, sino, más bien, lo que llamamos: el oficio, es decir, todo aquello que en el ejercicio sostenido de su trabajo le ha permitido a cada uno enriquecerse, de modo que en lo sucesivo, en el oficio correspondiente, como dice muy bien nuestra locución, es alguien entendido en el asunto. Seguramente, este rasgo común es el que llamó la atención de los griegos, y no la diferencia que separa, a pesar de ello muy claramente -pero bajo la condición de mirar en una dirección muy diferente-, una mesa, por ejemplo, de una estatua de Poseidón.

Veamos esto un poco más de cerca. Una mesa es hecha, por ejemplo, para ser el soporte de los cubiertos, de la vajilla, en una palabra, de todo aquello que permite sentarse a la mesa para ingerir una comida. Pero ¿una estatua de Poseidón? Manifiestamente, no está ahí para algo, lo que sea -al menos, en el sentido preciso de la "utilidad" tal como la hemos circunscrito. Porque decir que la estatua de Poseidón está ahí para representar al dios sería un contrasentido. Ante todo, porque en la visión griega originaria la estatua en cuestión es el dios: ella lo hace aparecer a él mismo, en todo el esplendor de su majestad. No hay aquí ninguna necesidad de extenderse sobre ideas estrafalarias: los griegos no "creen" que ese bronce sería, por una suerte de transferencia fetichista, el dios del mar.

Al contrario, por la estatua ellos están directamente en relación con la modalidad de aparición del dios (¡una modalidad que también es extremadamente pasajera!). Con la obra, en consecuencia, vemos manifestarse tanto la no-utilidad de la cosa como la no-permanencia del útil. Pero hablar así es aún -inclusive si no es más que negativamente- tomar la cosa y el útil como referencias.

9 "La pregunta por la técnica", Filosofia, Ciencia y Técnica, Ed. Universitaria, Santiago de Chile, $5^{\mathrm{a}}$ ed., 2007. Trad. de Francisco Soler Grima. Edición de Jorge Acevedo Guerra. Véase también, F. Fédier: "Étude de la $\tau \dot{\chi} \chi v \eta$ [téchne]”; en L'art en liberté, ed. cit., pp. 40-90. 
Sin duda, no es posible hablar directamente de la obra, a menos que tengamos el hilo de Ariadna gracias al cual mantenemos contacto con su inverosímil inmediatez.

Aquí las artes plásticas, a pesar de lo que son, nos obnubilan. Por eso debo evocar otro arte. Por lo demás ¿se puede hablar aquí de otro arte? El solo nombre de la poesía - puesto que se trata de ella-indica claramente hasta qué punto los antiguos griegos entendían del asunto del arte. Poesía, en efecto, es en ellos el sinónimo estricto

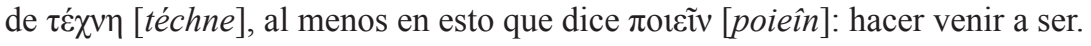

¿Cómo se presenta la poesía? "En Grecia, escribe Rimbaud, versos y liras ritman la Acción" $"$. Para entender esto enteramente es preciso, por ejemplo, pensar en Píndaro, cuyas Odas triunfales ${ }^{11}$ son una celebración de una solemnidad que para nosotros resulta casi irrepresentable. No solamente un texto, sino un texto cantado y acompañado por la lira y las flautas; no solamente un canto, sino un canto cuyas alternancias rítmicas eran subrayadas por la danza de un coro. Si agrego que la métrica de las odas es de un extremo refinamiento, y que todo, desde entonces, refleja ese refinamiento, se puede comenzar a entrever la obra poética como un acontecimiento al cual el hecho de asistir pide, de una manera o de otra, que se tome parte en él.

Las mismas observaciones pueden ser hechas a propósito de esta otra cara de la poesía que es, al lado de la poesía lirica, la tragedia. Ahí también, no solamente poema, sino danza (ahí también bajo una forma eminentemente coreográfica, es decir, codificada). En pocas palabras: la poesía es música, del mismo modo que la música es poesía. Es ahí donde podemos entrever, en la integridad de su contenido real, la íntima relación entre la obra y el tiempo. Decía antes que estamos en el mundo de las obras de una manera efímera. Ahora es preciso traer a colación la precisión decisiva: efímero no significa que estemos allí solo por "poco tiempo" -mientras que, frente a eso, estaríamos "todo el tiempo" en el mundo de las cosas. El tiempo verdadero como también el espacio verdadero no son eso que habitualmente creemos que son. El tiempo verdadero tiene lugar -literalmente- de cuando en cuando; de la misma manera, el espacio no se abre sino cuando hay espaciamiento del espacio. Son las obras las que hacen ser al tiempo verdadero y al espacio verdadero.

Así hemos llegado ante la última observación por hacer esta tarde. Concierne al título completo de la conferencia: El Origen de la obra de arte.

Tomamos habitualmente la palabra origen entendiéndola así: aquello de donde proviene algo - sin preguntarnos, más allá de eso, sobre la modalidad de esta proveniencia.

10 “Segunda carta: De Arthur Rimbaud a Paul Demeny, 15 de mayo de 1871”, Una temporada en el infierno, seguido de Iluminaciones, seguido de Cartas del vidente. Introducción, traducción y notas de Ramón Buenaventura. En la página electrónica de homenaje de Ramón Buenaventura al joven poeta Arthur Rimbaud; consultada: junio de 2015 (http://rimbaud. rbuenaventura.com). Véase, también: M. Heidegger: "Rimbaud vivant", Experiencias del pensar (1910-1976), Ed. Abada, Madrid, 2014. Trad. de Francisco de Lara.

11 Píndaro. Odas y fragmentos, Biblioteca Básica Gredos, Madrid, 2002. Introducción general de Emilia Ruiz Yamuza. Trad. y notas de Alfonso Ortega. 
Así se nos escapa el secreto del origen, a saber, que no puede dar a luz a menos que él mismo desaparezca. Dicho de otra manera: no hay "origen" si no es a favor de eso que ha sido originado, sin una desviación que, en adelante, va a separar lo originado del origen. Esta desviación puede, inclusive, tomar la figura de una liberación, en la cual, en adelante, se juega toda posibilidad tanto de conservar el origen como, ante todo, de tener una relación con él.

El origen de la obra de arte, dice Heidegger, es la verdad. Apenas avanzaremos mientras tengamos una idea vaga y convencional de la verdad. Esta última no es sino la definición tradicional de la verdad como adecuación, y más precisamente: adecuación de lo que nosotros decimos - 0 , más en general, de nuestra representación- a lo que es en realidad.

No es de esta verdad de la que habla Heidegger. Él invita a escuchar el nombre griego de la verdad, y a entender lo que dice ese nombre. 'A $\lambda \hat{\theta} \theta \varepsilon 1 \alpha$ [Alétheia]-todavía es necesario repetirlo, más de sesenta años después de que Heidegger lo publicó por primera vez; esta palabra habla directamente a todo oído que sepa un rudimento de griego, y le hace reparar en el alfa privativa y el tema que deriva del verbo $\lambda \alpha v \theta \alpha ́ v \omega$ [lantháno]: escapar.

Lo que dice $\dot{\alpha} \lambda \eta \dot{\theta} \theta \varepsilon 1 \alpha$ [alétheia] es tan simple como directo: la verdad, tal como se ha dicho aquí, es esta situación precisa donde el movimiento de escapar es interrumpido. La experiencia griega de la verdad está completamente gobernada por el sentimiento propiamente trágico en que se encuentra el ser humano, quien debe rendirse a la evidencia de que todo lo que sabe o cree saber es vacilante, siempre a punto de escapársele, pero para quien, de cuando en cuando, esta escapada es interrumpida, y a quien, en el lapso de un relámpago, hace frente lo que es.

Esta verdad, que no aparece sino para desaparecer enseguida -lo que no implica en modo alguno que no podamos guardarla en la memoria-, es lo que, según Heidegger, constituye el origen de la obra de arte. Toda obra de arte, podemos agregar, es precisamente una de las modalidades en las que se guarda en la memoria el relampaguear.

Evidentemente, habría aún muchas cosas que decir después de esta exposición, de la que no me oculto su carácter demasiado abrupto. Pero, como les he dicho al comenzar, deseo verdaderamente que, juntos, podamos ahora plantearnos preguntas que -así lo espero- hayan brotado en nosotros. Estas preguntas conciernen a la obra de arte y a su origen. Tal vez la manera más simple de ponernos en una buena disposición para entablar nuestra conversación consiste en dejar la primera palabra a un poeta. René Char ¿no habla a partir del origen de la obra de arte cuando enuncia cómo se ha confiado a su lealtad? Oigamos bien el acento optativo de su declaración:

\section{L'éclaire me dure}

\section{El relámpago me parece largo}


("L'éclaire me dure"; en La bibliothèque est en feu (La biblioteca en llamas), Pléiade, p. 378).

El texto XXIV de À la santé du serpent (A la salud de la serpiente), Pléiade, p. 266, abre un horizonte de comprensión; dice:

"Si nous habitons un éclair, il est le cœur de l'eternel".

"Si nosotros habitamos un relámpago, él es el corazón de lo eterno".

Traducción de Jorge Acevedo Guerra Universidad de Chile joaceved@gmail.com 